\title{
DECONFINEMENT AT FINITE BARYON NUMBER DENSITY
}

\author{
J. ENGELS and H. SATZ \\ Fakultät für Phisik. Linitersitäı Bielefeld. Bielefeld. Germany.
}

Received 21 May 1985

\begin{abstract}
We study the deconfinement transition for non-vanishing baryonic chemical potential $\mu$. on an $8^{3} \times 3$ lattice. with Wilson fermions in lowest-order hopping parameter expansion. For very light quarks. we find that the relative deconfinement temperature $T_{c}(\mu) / T_{c}(0)$ has decreased by more than 20 , when $\mu / T_{c}(0)=1$.
\end{abstract}

Deconfinement in a system with non-vanishing baryon number density poses one of the most interesting open problems in statistical quantum chromodynamics. It is also a rather urgent problem, since forthcoming nuclear collision experiments to study deconfinement will certainly provide baryon-rich systems.

In the present note, we want to present some first results obtained in studying the effect of non-vanish. ing baryonic chemical potential $\mu$ on the deconfinement temperature $T_{\mathrm{c}}$. They already give us some indication of what the complete phase diagram for strongly interacting matter looks like.

The pattern of deconfinement in the presence of dynamical quarks was for some time rather unclear even for $\mu=0$, since the fermion field breaks the global symmetry used to characterize the two phases in pure gauge theory. However, the deconfinement phenomenon has been found to persist, at least as a very abrupt change of regimes, in a variety of different fermion schemes ${ }^{\ddagger 1}$; it appears that the symmetry breaking induced by the quarks is in fact very small. This is in accord with the interpretation of deconfinement as the QCD form of an insulator-conductor transition, where string breaking provides a non-vanishing but small "colour conductivity" in the confinement region [2].

The basis of statistical QCD is the partition function

${ }^{\ddagger 1}$ For a recent review see ref. [1].
$Z(T, \mu)=\operatorname{Tr} \exp [-(H-\mu N) / T]$,

where $H$ is the QCD hamiltonian and $N$ the net quark number operator. To obtain a form suitable for computer simulation, we want to rewrite this in the form of a sum over field configurations on the lat tice. The resulting formulation has been obtained by several authors [3-6]. Writing the fermion action as sum over quark flavours $f$ in the Wilson formulation [7]

$S_{\mathrm{Q}}=\sum_{f} \bar{\psi}_{f}(1-\kappa M) \psi_{f}$,

where $\kappa$ denotes the hopping parameter, we have

$$
\begin{aligned}
& M_{n m}=\sum_{\nu=1}^{3}\left[\left(1-\gamma_{\nu}\right) U_{n m} \delta_{n, m-\hat{\mathbf{v}}}\right. \\
& \left.\quad+\left(1+\gamma_{\nu}\right) U_{m n}^{+} \delta_{n, m+\hat{\mathbf{v}}}\right] \\
& \quad+f(\mu a)\left(1-\gamma_{0}\right) U_{n m} \delta_{n, m-\hat{\mathbf{0}}} \\
& \quad+[1 / f(\mu a)]\left(1+\gamma_{0}\right) U_{m n}^{+} \delta_{n, m+\hat{0}}
\end{aligned}
$$

The notation is that of ref. [2]: $\psi_{n}$ denotes the fermion field at site $n, U_{n m}$ the SU(3) matrix on the link between the adjacent sites $n$ and $m$, and $\hat{\mathbf{v}}$ is a unit vector from the given site in the $\nu$ direction. We consider quarks of vanishing bare mass; spatial and temporal lattice spacings are set equal. The modification due to non-zero chemical potential $\mu$ is contained in $f(\mu a)$; following refs. $[3,4]$, we choose

$f(\mu a)=\exp (\mu a)$, 
where $a$ denotes the lattice spacing; for a more general form, see ref. [6]. After integration of the fermion variables, we have the partition function in the form

$$
\begin{aligned}
& Z(T, \mu)=\int \prod_{\text {link s }} \mathrm{d} U[\operatorname{det}(1-\kappa M)]^{N_{\mathrm{f}}} \\
& \quad \times \exp \left[-S_{\mathrm{G}}(U)\right],
\end{aligned}
$$

where $N_{\mathrm{f}}$ counts the number of quark flavours and $S_{\mathrm{G}}$ is the usual Wilson action for the gauge field sector,

$$
\begin{gathered}
S_{\mathrm{G}}(U)=\frac{6}{g^{2}}\left(\sum_{P_{\sigma}}\left(1-\frac{1}{3} \operatorname{Re} \operatorname{Tr} U U U U\right)\right. \\
\left.+\sum_{P_{\beta}}\left(1-\frac{1}{3} \operatorname{Re} \operatorname{Tr} U U U U\right)\right),
\end{gathered}
$$

expressed in terms of space-space and space-time plaquette sums.

The main problem in the evaluation of relation (5), and all quantities derived from it, is that the effective fermion action

$S_{\mathrm{F}}=-N_{\mathrm{f}} \log [\operatorname{det}(1-\kappa M)]$

is complex and hence cannot be used as weight for updating the lattice. To see this explicitly, consider the hopping parameter expansion [8]

$\log \operatorname{det}(1-\kappa M)=-\operatorname{Tr} \sum_{l=1}^{\infty} \frac{\kappa^{l}}{l} M^{l}$.

Using eqs. (3), (4), it yields for lattices with $N_{\beta} \leqslant 3$ temporal lattice sites as leading term

$$
\begin{aligned}
S_{\mathrm{F}}^{\mathrm{L}} & =-2 N_{\mathrm{f}}(2 \kappa)^{N_{\beta}} \sum_{\boldsymbol{x}}\left[L(x) \exp \left(N_{\beta} \mu a\right)\right. \\
& \left.+L^{*}(\boldsymbol{x}) \exp \left(-N_{\beta} \mu a\right)\right],
\end{aligned}
$$

here

$L(x) \equiv \operatorname{Tr}\left(\prod_{\tau=1}^{N_{\beta}} U_{x ; \tau, \tau+1}\right)$

is the Polyakov loop at the spatial site $x$. Eq. (5) can be rewritten in the form

$$
\begin{aligned}
S_{\mathrm{F}}^{\mathrm{L}} & =-4 N_{\mathrm{f}}(2 \kappa)^{N_{\beta}} \sum_{x}\left[\operatorname{Re} L(x) \cosh N_{\beta} \mu a\right. \\
& \left.+\mathrm{i} \operatorname{Im} L(x) \sinh N_{\beta} \mu a\right]
\end{aligned}
$$

which in general is real only for $\mu=0$.

A Monte Carlo evaluation becomes possible, how. ever, if we consider

$$
\begin{aligned}
& Z(T, \mu)=\int \prod_{\text {links }} \mathrm{d} U\left[\exp \left(-\mathrm{i} \operatorname{Im} S_{\mathrm{F}}\right)\right] \\
& \quad \times \exp \left(-S_{\mathrm{G}}-\operatorname{Re} S_{\mathrm{F}}\right),
\end{aligned}
$$

using only the real part of the fermion action, togeth. er with the gluon action, for the weight in the link updating procedure. For $\mu=0, \operatorname{Im} S_{\mathrm{F}}=0$, and so we expect some range of $\mu$ where $\operatorname{Im} S_{\mathrm{F}}$ does not yet contribute very much. We can therefore obtain a first idea of the effect of finite baryon number density on deconfinement by "quenching" the imaginary part of $S_{\mathrm{F}}$. This partial quenching, in contrast to a complete quenching with $\operatorname{det}(1-\kappa M)=1$ in eq. (5), leaves a $\mu$-dependence in all thermodynamic observables.

The results we shall present here are obtained in leading order hopping parameter expansion, using the partial quenching approximation. We work with two quark flavours on an $8^{3} \times 3$ lattice, where

$\operatorname{Re} S_{\mathrm{F}}^{\mathrm{L}}=-h \sum_{\boldsymbol{x}} \operatorname{Re} L(\boldsymbol{x})$,

with

$h \equiv 8(2 \kappa)^{3} \cosh 3 \mu a$.

We note that in this approximation the introduction of a chemical potential has the same effect as shifting the hopping parameter $k$ to a larger value. This is known [9] to result in a decrease of the critical coupling and hence of the deconfinement temperature. We thus already know that the effect is qualitatively as expected.

In table 1 we list our Monte Carlo results for four different values of $h$. Shown in the first column is the associated $\kappa$ value if $\mu=0$. The critical coupling val-

Table 1

Critical coupling and temperature at deconfinement.

\begin{tabular}{llll}
\hline$\kappa(\mu=0)$ & $h$ & $\left(6 / g_{\mathrm{c}}^{2}\right)$ & $T_{\mathrm{c}} / \Lambda_{\mathrm{L}}^{N_{\mathrm{f}}=2}$ \\
\hline 0.120 & 0.111 & 5.390 & 170 \\
0.140 & 0.176 & 5.367 & 165 \\
0.156 & 0.243 & 5.277 & 147 \\
0.180 & 0.373 & 5.101 & 117 \\
\hline
\end{tabular}


Table 2

Baryon chemical potential at deconfinement.

\begin{tabular}{llllc}
\hline$\kappa(\mu=0)$ & $m_{\mathrm{q}} / T_{\mathrm{c}}$ & $h$ & $\mu_{\mathrm{c}} a$ & $\mu_{\mathrm{c}} / \Lambda_{\mathrm{L}}^{N_{\mathrm{f}}=2}$ \\
\hline \multirow{2}{*}{0.12} & \multirow{2}{*}{2.00} & 0.111 & 0 & 0 \\
& & 0.176 & 0.346 & 172 \\
& & 0.243 & 0.475 & 210 \\
0.14 & 0.87 & 0.176 & 0 & 0 \\
& & 0.243 & 0.283 & 125 \\
0.156 & 0.10 & 0.243 & 0 & 0 \\
& & 0.373 & 0.331 & 117 \\
\hline
\end{tabular}

ues in the third column correspond to the point of maximal change in the energy density, i.e., to the peak in the specific heat of deconfinement. The last column lists the critical temperature $T_{\mathrm{c}}=\left[3 a\left(g_{\mathrm{c}}\right)\right]^{-1}$ obtained through the renormalization group relation for $N_{\mathrm{f}}=2$,

$$
\begin{aligned}
& a \Lambda_{\mathrm{L}}=\exp \left\{-\frac{4}{29} \pi^{2}\left(6 / g^{2}\right)\right. \\
& \left.\quad+\left(345 / 29^{2}\right) \log \left[\frac{8}{29} \pi^{2}\left(6 / g^{2}\right)\right]\right\},
\end{aligned}
$$

the decrease with increasing $\kappa$ or $h$ is clearly seen.

For fixed $\kappa$, different $h$ correspond to different values of the critical chemical potential $\mu$, as given by eq. (14). In table 2 , we list the resulting $\mu_{\mathrm{c}}$ for a given choice of $\kappa$. As in table 1 , the values of $\mu_{\mathrm{c}} / \Lambda_{\mathrm{L}}^{N_{\mathrm{f}}=2}$ are obtained by use of relation (15). In the hopping parameter formulation, the value of $k$ used determines an effective quark mass $m_{\mathrm{q}}$ in the lattice action. Requiring $m_{\mathrm{q}}=0$ defines a limiting value $\kappa_{\mathrm{ch}}$, at which the action becomes chirally symmetric; it has been calculated both numerically and in the weak coupling limit (see ref. [2] for further details and references). The quark mass $m_{\mathrm{q}}$ for a given $\kappa<\kappa_{\mathrm{ch}}$ is expected to be [10]

$\frac{1}{2}\left(1 / \kappa-1 / \kappa_{\mathrm{ch}}\right)=\exp \left(m_{\mathrm{q}} a\right)-1$.

The values of $m_{\mathrm{q}} / T_{\mathrm{c}}(\mu=0)$ resulting from eq. (16) are also listed in table 2.

In fig. 1 we show the deconfinement phase diagram as it emerges from these results; the curves are drawn just to guide the eye. For the largest quark mass considered, $T_{\mathrm{c}}(\mu)$ is rather independent of $\mu$ up to about $\mu / T_{c}(0) \simeq 1$, while for lighter quarks the $\mu$ dependence sets in sooner. Such a behaviour is in fact expected:

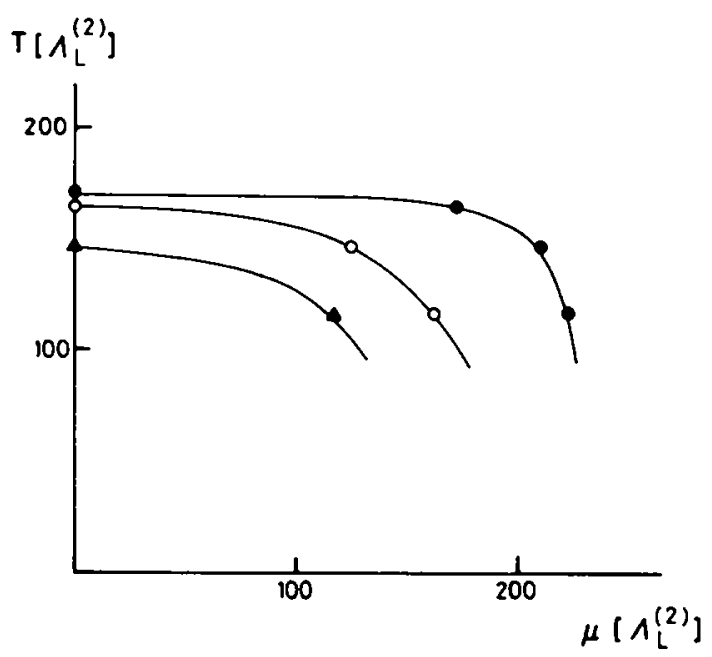

Fig. 1. Phase diagram for strongly interacting matter, for dynamical quarks of mass $m_{\mathrm{q}} / T_{\mathrm{c}}(0)=2.00(\bullet), 0.87(0)$ and $0.10(4)$.

heavy quarks are thermodynamically suppressed, and so for small $\mu$, the system is essentially gluonic; hence $T_{\mathrm{c}}$ is independent of $\mu$. Sufficiently large $\mu$, however, with a correspondingly large quark number density, force the fermion sector to become important for the thermodynamics; hence now $T_{\mathrm{c}}$ drops with increasing $\mu$. On the other hand, for $m_{\mathrm{q}} \sim 0$, quark and gluon sectors are on equal footing even at $\mu=0$, so here the $\mu$-dependence of $T_{\mathrm{c}}$ sets in as soon as $\mu$ is turned on. First studies of chiral symmetry restoration in baryonrich environments have given indications of similar behaviour there [4].

In conclusion, we note that our results give an encouraging first glance at the deconfinement phase structure of strongly interacting matter. The results are approximative on two accounts: the imaginary part of the fermion action is neglected, and only the leading term of the hopping parameter expansion is considered. We are presently extending our calculations to include both $\operatorname{Im} S_{\mathrm{F}}$ and higher order terms in $\kappa$, to obtain a quantitative measure of the approxima. tions used here.

\section{References}

[1] E.g. H. Satz, in: Quark matter '84, ed. K. Kajantie (Springer, Berlin, 1985).

[2] T. Çelik, J. Engels and H. Satz, Bielefeld preprint BI-TP $84 / 09$ (1984), Nucl. Phys. B [FS], to be published. 
[3] P. Hasenfratz and F. Karsch, Phys. Lett. 125B (1983) 308.

[4] J. Kogut et al., Nucl, Phys. B225 [FS9] (1983) 93.

[5] N. Bilić and R.V. Gavai, Z. Phys. C23 (1984) 77.

[6] R.V. Gavai, Brookhaven preprint BNL 36267 (1985), Phys. Rev. D., to be published.

[7] K. Wilson, Phys. Rev. D10 (1974) 145; and in: New phenomena in subnuclear physics (Erice, 1975), ed. A. Zichichi (Plenum, New York, 1977).
[8] A. Hasenfratz and P. Hasenfratz, Phys. Lett. 104B (1981) 489;

C. Lang and H. Nicolai, Nucl, Phys. B200 [FS4] (1982) 135.

[9] T. Celik, J. Engels and H. Satz, Phys. Lett. 133B (1983) 427.

[10] A. Hasenfratz, P. Hasenfratz, Z. Kunszt and C.B. Lang, Phys. Lett. 110B (1982) 289. 